\title{
Same day colposcopic examination and loop electrosurgical excision procedure (LEEP) presents minimal overtreatment and averts delay in treatment of cervical intraepithelial neoplasia in Kenyatta National Hospital, Kenya"
} \author{
Kosgei J. Rose ${ }^{3,4 \#}$ \\ ${ }^{1}$ Ministry of Medical Services, Kabarnet District Hospital, Baringo, Kenya \\ ${ }^{2}$ Kenya Medical Research Institute, Nairobi, Kenya \\ ${ }^{3}$ Department of Obstetrics and Gynecology, University of Nairobi, Nairobi, Kenya \\ ${ }^{4}$ Kenyatta National Hospital, Department of Research and Special Programs, Nairobi, Kenya \\ Email: $\underline{\text { salilkabon@yahoo.com }}$
}

Kays Muruka ${ }^{1}$, Mugo R. Nelly ${ }^{2,3}$, Wanyoike Gichuhi, ${ }^{3,4}$ Kihara Anne-Beatrice $^{3,4}$, Cheserem J. Eunice ${ }^{3,4}$,

Received 23 February 2013; revised 24 March 2013; accepted 2 April 2013

Copyright (C) 2013 Kays Muruka et al. This is an open access article distributed under the Creative Commons Attribution License, which permits unrestricted use, distribution, and reproduction in any medium, provided the original work is properly cited.

\begin{abstract}
Background: Screening for cancer of the cervix at Kenyatta National Hospital (KNH), follows the recommended three-step strategy; Papanicolaou (Pap) smear, colposcopy/biopsy and loop electrosurgical excision procedure (LEEP)/biopsy. This approach poses the following challenges: multiple clinic visits, costly, time consuming, long turnaround time to treatment, non-compliance and loss-to-follow-up. Objective: To determine the agreement between histologies following colposcopy and LEEP amongst women in KNH as a forerunner for opportunity to shift from the three-step approach to the two-step "see and treat” (same-day colposcopy and LEEP) approach. Methods: This was a retrospective descriptive cohort of Women who underwent LEEP procedure between January 2008 and 31st December 2010 following the three-step approach at KNH, Kenya. Results: A total of 124 patients out of the 132 patients who underwent LEEP were included in the analysis. The 8 patients excluded had missing files. HIV infected, uninfected or unknown women are similar socio-demographically. The mean (SD) age for the HIV infected, uninfected and unknown is 37 (6), 33 (10) and 35 (9) years

\footnotetext{
*Meetings at which parts of the data were presented: 3rd inter-CFAR symposium on HIV research in women, September 2012, Providence Rhode Island, USA. Part of the data analyzed were presented by $\mathrm{Mu}-$ ruka Kays for his Master in Medicine in Obstetrics and Gynaecology of University of Nairobi, 2011.

\#Corresponding author.
}

respectively. Colposcopic and LEEP biopsy histology within patients demonstrated a high weighted kappa statistics agreement of $84 \%$. LEEP increased diagnosis of invasive cancer. Patients had a median (IQR) 5 (4 - 6) clinic visits from Pap smear to LEEP treatment. It took median (IQR) 55 (27 - 116) days between Pap smear to colposcopy result and $167(101$ - 276) days between Pap smear results to LEEP treatment. If a LEEP procedure were to be performed in this cohort of women on the same day of the colposcopy biopsy a median (IQR) 77 (55 - 137) days could have been saved. Conclusion: There is a high agreement between colposcopy and LEEP biopsies in our setting offering a window of opportunity to perform "See and Treat” same-day colposcopy and LEEP treatment procedure, skipping the colposcopy biopsy stage.

Keywords: Colposcopy; Loop Electrosurgical Excision Procedure (LEEP); CIN; Cervical Cancer Screening

\section{INTRODUCTION}

Timely detection and treatment of cervical intraepithelial neoplasia (CIN) whose aetiopathogenesis is oncogenic sub-types of Human Papilloma Virus (HPV); is key in abating invasive cancer of the cervix [1,2]. This is important especially in low resource settings and amongst HIV-infected patients where it has a more aggressive progression to invasive cancer [3-5]. Screening for cancer 
of the cervix at Kenyatta National Hospital (KNH), follows the three-step strategy recommended by the American Society for Colposcopy and Cervical Pathology (ASCCP) guidelines, which entails Papanicolaou (Pap) smear, colposcopy/biopsy and Loop electrosurgical excision procedure (LEEP)/biospy [6]. Challenges presented by this approach in resource constraint settings to the patient include: multiple clinic visits, costly, time consuming, protracted duration from diagnosis to definitive treatment with poorer prognosis, psycho-social sequelae while awaiting biopsy results, non-compliance to treatment and loss-to-follow-up [6]. To the health system, glaring challenges are limited number of pathologists and colposcopists hence the need for innovative strategies to optimize treatment of $\mathrm{CIN}$ by reducing contact visits to treatment [7].

The "See and Treat" approach is a one-step management with immediate treatment of CIN lesions by sameday colposcopy and LEEP biopsy (LEEP is an office procedure done under local anesthesia where cone excision of CIN lesion surrounding the endocevical canal and transformation zone of the cervix is done by use of a thin low-voltage electrified wire loop). This skips the colposcopy directed biopsy stage and has several benefits over the traditional standard of care because of its ability to provide histological diagnosis and treatment of CIN simultaneously at the same sitting. The advantages include reduction of patient clinic visits, reduction of time interval from diagnosis to treatment, more accurate histological diagnosis due to a larger specimen for evaluation unlike in colposcopic biopsy, decrease in cost, patient convenience with improved compliance and enhanced follow-up [8]. The major critique towards this approach is the risk of overtreatment or unnecessary treatment amongst patients with low grade squamous intraepithelial lesions (LSIL) and physiological variants (cervical ectopy and metaplasia). These have been attributed to intra and inter-observer variability [6]. The National Health service Cervical Screening Programme (NHSCSP), 2010 guidelines state that overtreatment should be periodically audited and be set at a standard cut-off of less than $10 \%$ which is consistent with the recommendation of the Cochrane Colposcopy and Cervical Cytopathology Collaborative [9-11]. NHSCSP guidelines, 2010 showed that overtreatment was $68 \%$ among patients with LSIL and only $7 \%$ in patients with high grade squamous intraepithelial (HSIL) [11]. Therefore in patients with HSIL overtreatment should not be a deterrent to the "See and treat" approach. Cervical screening programs should consider revising their CIN treatment algorithms to include patients with HSIL for same day colposcopy and LEEP biopsy.

We carried out an operations research study to determine the agreement between histologies following col- poscopy and LEEP amongst women in Kenyatta National Hospital as a forerunner for opportunity to shift from the three-step approach to the two-step "see and treat" (same-day colposcopy and LEEP) approach.

\section{METHODS}

\subsection{Study Design}

This was a retrospective descriptive cohort study.

\subsection{Study Site and Setting}

The study was conducted at Kenyatta National Hospital $(\mathrm{KNH})$, the largest public referral hospital in Kenya and the teaching Hospital for the University of Nairobi Medical School. The catchment of KNH is largely made up of patients of low socio-economic status. Treatment protocols for women with a cytological diagnosis of $\geq$ HSIL or recurrent LSIL are triaged to colposcopic examination and biopsy. Those with a confirmation of CIN $2 / 3$ are treated with LEEP and reviewed with histopathology results four weeks later.

Since this is a care program different, colposcopists and pathologist participate in the care of these patients. The cost of colposcopy is 2500 Kenyan shillings (30 USD) and LEEP is 3200 Kenyan shillings (40 USD). In addition to these costs the patient has to cater for a minimum four clinic visits transportation costs (book for colposcopy, colposcopy procedure, interpretation of colposcopy result/book for LEEP and LEEP procedure), on occasion a patient may be required to have more visits if either histology result is not available during the clinic visit. All costs are paid by patients.

\subsection{Study Population}

Data retrieval of files for patients treated with outpatient LEEP from January 2008 to 31 st December 2010 at KNH. All records with both colposcopy directed biopsy and LEEP biopsy histology result were eligible for the study.

\subsection{Sample Size}

132 patients records met eligibility criteria.

\subsection{Data Collection and Management}

Data was retrospectively collected from routine patient case files. No patient identifiers were collected. The following variables were collected: age, marital status, education level, availability of phone contact, referral status, parity, HIV status, turn-around time (Pap smear to colposcopy and Pap smear to LEEP), number of clinic visits, Pap smear cytology, colposcopy histology and LEEP histology. The data was abstracted into an Excel database. 


\subsection{Data Analysis}

Analysis was done using STATA software. Continuous variables are summarised as mean (standard deviation [SD]) or median (inter-quartile range [IQR]). Categorical variables are summarised as frequency and percentages and their association and the HIV status was tested using a chi-square test. Level of agreement between colposcopic histology and LEEP histology is presented as weighted kappa statistics with more weight given to category disagreements that are far apart for instance normal versus invasive cancer. Days deemed saved by same day Colposcopy-LEEP, was calculated by getting the difference in days per patient by subtracting the "pap to colposcopy" days from "pap to LEEP days", these values were then summarized and the median (IQR) is reported. The conventional P-value cut-offs are used to assess the level of significance.

\subsection{Ethical Consideration}

Scientific and ethical approval for this study was obtained and approved by the Kenyatta National Hospital/ University of Nairobi Institutional Research and Ethics Committee. This study was retrospective and was classified as audit hence no participants were interviewed.

\section{RESULTS}

A total of 124 patients (49 HIV-infected, 22 HIV uninfected and 53 HIV unknown) out of the 132 patients who underwent loop electrosurgical procedure (LEEP) at Kenyatta National Hospital between 2008 and 2010 were eligible for this analysis. Eight patients had their files missing and were not included, Figure 1.

Among 124 women treated with LEEP at KNH, there were no differences in age, marital status, education level, parity or point of referral (Table 1).

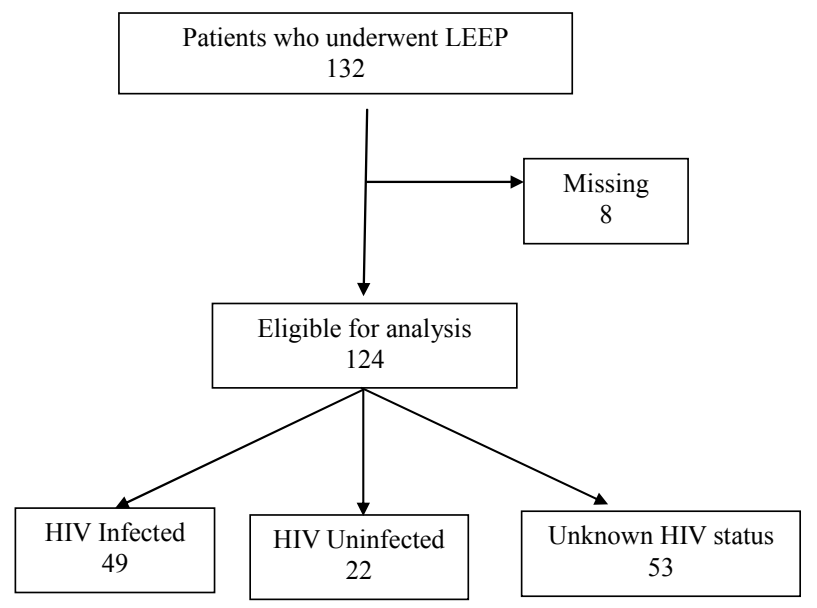

Figure 1. Eligibility flowchart for women who underwent LEEP procedure at Kenyatta National Hospital 2008-2010.
Table 1. Socio-demographic characteristics for women who underwent LEEP at Kenyatta National Hospital 2008-2010.

\begin{tabular}{|c|c|c|c|c|}
\hline \multirow[b]{2}{*}{ Variable } & \multicolumn{3}{|c|}{ HIV status } & \multirow[b]{2}{*}{ P-value } \\
\hline & $\begin{array}{c}\text { Infected } \\
(\mathrm{N}=49) \mathrm{n} \\
(\%)\end{array}$ & $\begin{array}{c}\text { Uninfected } \\
(\mathrm{N}=22) \mathrm{n} \\
(\%)\end{array}$ & $\begin{array}{c}\text { Unknown } \\
(\mathrm{N}=53) \mathrm{n} \\
(\%)\end{array}$ & \\
\hline Age Mean (SD) & $37(6)$ & $33(10)$ & $35(9)$ & 0.102 \\
\hline \multicolumn{5}{|l|}{ Marital status } \\
\hline Single & $12(24)$ & $6(27)$ & $17(32)$ & \multirow{5}{*}{0.146} \\
\hline Married & $32(65)$ & $12(55)$ & $27(51)$ & \\
\hline Separated/divorced & $2(4)$ & $2(9)$ & $4(8)$ & \\
\hline Widowed & $3(6)$ & $0(0)$ & $3(6)$ & \\
\hline Unknown & $0(0)$ & $2(9)$ & $2(4)$ & \\
\hline \multicolumn{5}{|l|}{ Education level } \\
\hline None/nursery & $3(6)$ & $0(0)$ & $3(6)$ & \multirow{5}{*}{0.597} \\
\hline Primary & $19(39)$ & $7(32)$ & $13(25)$ & \\
\hline Secondary & $18(37)$ & $8(36)$ & $16(30)$ & \\
\hline College & $5(10)$ & $4(18)$ & $16(30)$ & \\
\hline Unknown & $4(8)$ & $3(14)$ & $5(9)$ & \\
\hline \multicolumn{5}{|l|}{$\begin{array}{c}\text { Phone number } \\
\text { available }\end{array}$} \\
\hline Available & $41(84)$ & $18(82)$ & $44(83)$ & \multirow[t]{2}{*}{0.847} \\
\hline Not available & $8(16)$ & $4(18)$ & $9(17)$ & \\
\hline \multicolumn{5}{|l|}{ Referral status } \\
\hline $\mathrm{KNH}$ & $2(4)$ & $0(0)$ & $16(30)$ & \multirow{4}{*}{0.805} \\
\hline Other within Nairobi & $18(37)$ & $9(41)$ & $1(2)$ & \\
\hline Other outside Nairobi & $25(51)$ & $11(50)$ & $31(58)$ & \\
\hline Unknown & $4(8)$ & $2(9)$ & $5(9)$ & \\
\hline Parity median (IQR) & $2(2-3)$ & $2(1-3)$ & $2(1-3)$ & 0.366 \\
\hline
\end{tabular}

Pap smear cytology, colposcopic and LEEP histology results are similar among HIV infected, uninfected and unknown women who underwent LEEP procedure, Table 2.

Colposcopic and LEEP biopsy histology within patients demonstrated a high weighted kappa statistics agreement of $84 \%$. LEEP increased diagnosis of invasive cancer (Table 3).

Women who underwent LEEP procedure had a median (IQR) 5 (4 - 6) clinic visits from Pap smear to LEEP treatment.

It took a median (IQR) 55 (27 - 116) days between Pap smear to colposcopy result and 167 (101 - 276) days between Pap smear results to LEEP treatment for women who underwent LEEP procedure. If a LEEP procedure were to be performed in this cohort of women on the same day of the colposcopy biopsy a median (IQR) 77 (55 - 137) days could have been saved, Table 4.

\section{DISCUSSION}

Data from this study demonstrate that there is a high agreement $(84 \%)$ between colposcopic biopsy and LEEP 
Table 2. Pap smear cytology, colposcopic and LEEP histology: women who underwent LEEP procedure at Kenyatta National Hospital 2008-2010.

\begin{tabular}{|c|c|c|c|c|}
\hline \multirow[b]{2}{*}{ Variable } & \multicolumn{3}{|c|}{ HIV status } & \multirow[b]{2}{*}{ P-value } \\
\hline & $\begin{array}{c}\text { Infected } \\
(\mathrm{N}=49) \\
\mathrm{n}(\%)\end{array}$ & $\begin{array}{c}\text { Uninfected } \\
\text { (N = 22) } \\
\text { n (\%) }\end{array}$ & $\begin{array}{c}\text { Unknown } \\
\left(\begin{array}{c}\text { N = 53) } \\
\text { n (\%) }\end{array}\right.\end{array}$ & \\
\hline \multicolumn{5}{|l|}{ Pap smear } \\
\hline LSIL & $3(6)$ & $3(14)$ & $8(15)$ & \multirow{6}{*}{0.284} \\
\hline HSIL & $34(69)$ & $16(73)$ & $40(75)$ & \\
\hline ASCUS & $5(10)$ & $3(14)$ & $1(2)$ & \\
\hline Invasive cancer & $1(2)$ & 0 & 0 & \\
\hline Other & $2(4)$ & 0 & $3(6)$ & \\
\hline missing & $4(8)$ & 0 & $1(2)$ & \\
\hline \multicolumn{5}{|c|}{ Colposcopic histology } \\
\hline Normal & $1(2)$ & $0(0)$ & $0(0)$ & \multirow{6}{*}{0.083} \\
\hline CIN I & $4(8)$ & $1(5)$ & $4(8)$ & \\
\hline CIN 2 & $9(18)$ & $9(41)$ & $24(45)$ & \\
\hline CIN 3 & $33(67)$ & $11(50)$ & $23(43)$ & \\
\hline Invasive cancer & $1(2)$ & $1(5)$ & 0 & \\
\hline Inflammatory & $1(2)$ & 0 & $2(4)$ & \\
\hline \multicolumn{5}{|l|}{ LEEP histology } \\
\hline Missing & $6(12)$ & $1(5)$ & $2(4)$ & \multirow{7}{*}{0.300} \\
\hline Normal & $2(4)$ & $2(9)$ & $9(17)$ & \\
\hline CIN I & $3(6)$ & $2(9)$ & $15(28)$ & \\
\hline CIN 2 & $8(16)$ & $9(41)$ & $14(26)$ & \\
\hline CIN 3 & $21(43)$ & $6(27)$ & $2(4)$ & \\
\hline Invasive ca & $5(10)$ & $1(5)$ & $7(13)$ & \\
\hline Inflammatory & $4(8)$ & $1(5)$ & $4(8)$ & \\
\hline
\end{tabular}

Table 3. Agreement between colposcopic and LEEP histology for women who underwent LEEP procedure at Kenyatta National Hospital 2008-2010.

\begin{tabular}{ccccccc}
\hline & \multicolumn{5}{c}{ LEEP histology } \\
\cline { 2 - 7 } Colposcopy & Normal & CIN 1 & $\begin{array}{c}\text { CIN 2 } \\
\text { and 3 }\end{array}$ & $\begin{array}{c}\text { Invasive } \\
\text { Cancer }\end{array}$ & Others Total \\
\hline Normal & 1 & 0 & 0 & 0 & 0 & 1 \\
CIN 1 & 0 & 4 & 3 & 0 & 2 & 9 \\
CIN 2 and 3 & 5 & 10 & 68 & 6 & 20 & 109 \\
$\begin{array}{c}\text { Invasive } \\
\text { cancer } \\
\text { Others }\end{array}$ & 0 & 0 & 0 & 2 & 0 & 2 \\
Total & 6 & 0 & 2 & 0 & 1 & 3 \\
\hline
\end{tabular}

Agreement according to the weighted Kappa statistics

\begin{tabular}{cccccc}
\hline Agreement & $\begin{array}{c}\text { Expected } \\
\text { agreement }\end{array}$ & Kappa & $\begin{array}{c}\text { Standard } \\
\text { error }\end{array}$ & Z & P-value \\
\hline $84.00 \%^{* *}$ & $81.44 \%$ & 0.1419 & 0.041 & 3.46 & $<0.001$ \\
\hline
\end{tabular}

${ }^{+}$Disagreement is highlighted in grey; ${ }^{* *}$ Weighted kappa statistics.
Table 4. Pooled Turn-around time and days deemed saved by same day Colposcopy-LEEP for women who underwent LEEP procedure at Kenyatta National Hospital 2008-2010.

\begin{tabular}{cccc}
\hline & \multicolumn{2}{c}{ Pooled turn-around time } & Difference \\
\hline & $\begin{array}{c}\text { Pap smear to } \\
\text { Colposcopy }\end{array}$ & $\begin{array}{c}\text { Pap smear to } \\
\text { LEEP }\end{array}$ & Days saved \\
\hline Median (IQR) days & $55(27-116)$ & $167(101-276)$ & $77(55-137)$ \\
\hline
\end{tabular}

biopsy in our cohort of women in Kenyatta National Hospital, Kenya who followed a three-step protocol of Pap smear, colposcopy/biopsy and LEEP/biopsy. This high agreement presents a window of opportunity for "See and Treat" same-day Colposcopy and LEEP, skipping the colposcopy biopsy stage (two-step approach). Shifting our protocol from a three-step approach to a two-step approach in our setting, has a potential of increasing the diagnosis of invasive cancer, reducing clinic visits which are currently a median (IQR) 5 (4 - 6) visits and reducing the turnaround time to treatment by a median (IQR) 77 (55 - 137) days. The two-step approach has a limitation of overtreatment, which can be minimized by including only patients with HSIL on Pap smear cytology in the algorithm [6,11]. Even when overtreatment occurs, in resource limited settings such as ours where there is a higher risk of disease progression and lost-to-follow-up, overtreatment in this case can be regarded as a "lesser evil".

The same-day colposcopy and LEEP has been done successfully in Chiang Mai University Hospital, Thailand which has an overtreatment rate for patients with HSIL on Pap smear of 7\% [9] which is below the recommended rate of $10 \%$ by the NHSCSP, 2010 guidelines. [11] In the same hospital menopause was shown to be an independent predictor of overtreatment; this can be reduced by limiting such an approach to premenopausal women only [12]. Lessons from Thailand are useful in the design of treatment algorithms and eligibility for the same-day colposcopy LEEP approach.

This study has two main limitations that need to be factored when interpreting the results. First, possible variability in colposcopic and histological evaluations are due to different colposcopist and pathologist respectively. Second, the study has a substantial proportion (40\%) of women with unknown HIV status despite the role of HIV infection on aetiopathogenesis. This did not affect the analysis because HIV infected, uninfected and unknown are similar socio-demographically in our study, which is odd. This is odd because, most HPV infections resolve spontaneously and, in $3 \%-10 \%$ of women, the infection becomes persistent [13]. Persistent infection of cervical tissue with HPV is causally linked with the development of cervical carcinoma and its cytological precursors; CIN [14]. HIV-infected women, compared to 
their HIV-uninfected counterparts have an increased risk for infection with oncogenic HPV [15]. In addition, HIV-infected women are frequently infected with multiple HPV types, and have a higher chance of presenting with a persistent infection or progression of these lesions to invasive cancer. The incidence of cancer of the cervix in HIV-infected women is estimated to be $900 / 100,000$ compared to $10 / 100,000$ in their uninfected counterparts [16]. HIV infected women have a high prevalence of CIN ranging 50\% - 76\% [17,18]. For this reason cervical cancer programs should strive to have patients tested for HIV infection. Despite this limitation, the data we present show that it is possible to perform "See and Treat" same-day Colposcopy and LEEP in our setting.

\section{CONCLUSION}

There is a high agreement between colposcopy and LEEP biopsies in our setting offering a window of opportunity to perform "See and Treat" same-day colposcopy and LEEP treatment procedure, skipping the colposcopy biopsy stage. This approach has a potential advantage of reducing the number of clinic visits and the added advantage of increasing diagnosis of invasive cancer. There is a need to have clear protocols that include only patients with HSIL on Pap smears to reduce the risk of overtreatment. Results from this study can be generalized to other resource limited settings like Kenya. There is a need for prospective pragmatic hypothesis driven studies to assess the utilization and impact of this approach in resource limited settings.

\section{CONTRIBUTIONS}

- This study was design and implemented by Muruka Kays (MK) and Nelly Mugo (NM), Wanyoike Gichuhi (WG);

- MK was responsible for data collection and data entry

- MK, Anne-Beatrice Kihara (AK), Rose Kosgei (RK) were responsible for data analyses;

- MK, AK, RK and Eunice Cheserem (EC) prepared the initial draft of manuscript;

- All authors reviewed the draft manuscript and provided input for final version of the report.

\section{REFERENCES}

[1] Li, Z.G., Qian, D.Y., Cen, J.M., Chen, G.D. and Shu, Y.H. (2009) Three-step versus "see-and-treat" approach in women with high-grade squamous intraepithelial lesions in a low-resource country. International Journal of Gynecology \& Obstetrics, 106, 202-205.

doi:10.1016/j.ijgo.2009.04.011

[2] Bosch, F.X., Manos, M.M., Munoz, N., et al. (1995) Prevalence of human papillomavirus in cervical cancer: A worldwide perspective. Journal of the National Cancer
Institute, 87, 796-802. doi:10.1093/jnci/87.11.796

[3] De Andrade, A.C., Luz, P.M., Velasque, L., et al. (2010) Factors associated with colposcopy-histopathology confirmed cervical intraepithelial neoplasia among HIV-infected women from Rio De Janeiro, Brazil. PLoS One, 6, e18297. doi:10.1371/journal.pone.0018297

[4] Frisch, M., Biggar, R.J. and Goedert, J.J. (2000) Human papillomavirus-associated cancers in patients with human immunodeficiency virus infection and acquired immunodeficiency syndrome. Journal of the National Cancer Institute, 92, 1500-1510. doi:10.1093/jnci/92.18.1500

[5] Clifford, G.M., Polesel, J., Rickenbach, M., et al. (2005) Cancer risk in the Swiss HIV Cohort Study: Associations with immunodeficiency, smoking, and highly active antiretroviral therapy. Journal of the National Cancer Institute, 97, 425-432. doi:10.1093/jnci/dji072

[6] Cho, H. and Kim, J.H. (2009) Treatment of the patients with abnormal cervical cytology: A "see-and-treat" versus three-step strategy. Journal of Gynecologic Oncology, 20, 164-168. doi:10.3802/jgo.2009.20.3.164

[7] Goldie, S.J., Kuhn, L., Denny, L., Pollack, A. and Wright, T.C. (2001) Policy analysis of cervical cancer screening strategies in low-resource settings: Clinical benefits and cost-effectiveness. The Journal of the American Medical Association, 285, 3107-3115.

doi:10.1001/jama.285.24.3107

[8] Katz, I.T. and Wright, A.A. (2006) Preventing cervical cancer in the developing world. The New England Journal of Medicine, 354, 1110. doi:10.1056/NEJMp068031

[9] Aue-Aungkul, A., Punyawatanasin, S., Natprathan, A., Srisomboon, J. and Kietpeerakool, C. (2011) "See and treat" approach is appropriate in women with high-grade lesions on either cervical cytology or colposcopy. Asian Pacific Journal of Cancer Prevention, 12, 1723-1726.

[10] Kyrgiou, M., Tsoumpou, I., Vrekoussis, T., et al. (2006) The up-to-date evidence on colposcopy practice and treatment of cervical intraepithelial neoplasia: The cochrane colposcopy \& cervical cytopathology collaborative group (C5 group) approach. Cancer Treatment Reviews, 32, 516-523. doi:10.1016/i.ctrv.2006.07.008

[11] Luesley, D. and Leeson, S. (2010) Colposcopy and Programme Management: Guidelines for the NHS Cervical Screening Programme. 2nd Edition, Public Health England, London.

[12] Kietpeerakool, C., Srisomboon, J., Khunamornpong, S., Siriaunkgul, S. and Sukkawattananon, W. (2007) How can the overtreatment rate of "see and treat" approach be reduced in women with high-grade squamous intraepithelial lesion on cervical cytology? Asian Pacific Journal of Cancer Prevention, 8, 206-208.

[13] Monsonego, J., Bosch, F.X., Coursaget, P., et al. (2004) Cervical cancer control, priorities and new directions. International Journal of Cancer, 108, 329-333. doi:10.1002/ijc. 11530

[14] Cuschieri, K.S., Cubie, H.A., Whitley, M.W., et al. (2005) Persistent high risk HPV infection associated with development of cervical neoplasia in a prospective population study. Journal of Clinical Pathology, 58, 946-950. doi:10.1136/jep.2004.022863 
[15] Palefsky, J.M. (2003) Cervical human papillomavirus infection and cervical intraepithelial neoplasia in women positive for human immunodeficiency virus in the era of highly active antiretroviral therapy. Current Opinion in Oncology, 15, 382-388. doi:10.1097/00001622-200309000-00007

[16] Mandelblatt, J.S., Fahs, M., Garibaldi, K., Senie, R.T. and Peterson, H.B. (1992) Association between HIV infection and cervical neoplasia: Implications for clinical care of women at risk for both conditions. AIDS, 6, 173-178. doi:10.1097/00002030-199202000-00005
[17] Parham, G.P., Sahasrabuddhe, V.V., Mwanahamuntu, M.H., et al. (2006) Prevalence and predictors of squamous intraepithelial lesions of the cervix in HIV-infected women in Lusaka, Zambia. Gynecologic Oncology, 103, 1017-1022. doi:10.1016/j.ygyno.2006.06.015

[18] Moodley, J.R., Hoffman, M., Carrara, H., et al. (2006) HIV and pre-neoplastic and neoplastic lesions of the cervix in South Africa: A case-control study. BMC Cancer 6, 135. doi:10.1186/1471-2407-6-135 